\title{
Special issue: Cutting Edge of Scattering from Softmaterials
}

\author{
Kazuo Sakurai ${ }^{1} \cdot$ Mikihito Takenaka $^{2} \cdot$ Keiji Tanaka $^{3}$
}

Received: 30 October 2018 / Accepted: 30 October 2018 / Published online: 5 February 2019

(c) The Society of Polymer Science, Japan 2018

Observing structures and their dynamical behaviors are essential to understand polymers, but quite challenging because their spatial and time ranges are quite large: at least 3 or 4 orders of magnitude. Such observation is also a key in industry because controlling polymeric materials in their morphology determines performance in the final products. Synchrotron X-ray may be an only solution for these demands.

X-rays were discovered in 1895 by W.C. Röntgen and named "X-rays" because of their unknown nature. In 1912, M. von Laue and P. Knipping obtained the first X-ray diffraction pattern from a crystal, a ground-breaking discovery as a powerful tool to explore nanoscale structures of materials. Synchrotron X-rays were first observed in 1947 and soon began to be recognized as an exceptionally useful tool because of its bright light, more than $10^{6}$ times stronger than conventional X-rays. The construction of the thirdgeneration synchrotron facility and the brightest light source in the world: SPring-8 was initiated in Japan in 1991 and its operation was started in 1997. Among the various beamlines in SPring-8, a new beamline dedicated to polymers was constructed in 2009 for use in the simultaneous smalland wide-angle $\mathrm{X}$-ray scattering measurements, small- and wide-angle $\mathrm{X}$-ray scattering under grazing incidence, $\mathrm{X}$-ray diffraction, and X-ray reflectivity $[1,2]$.

This Polymer Journal special issue is aimed to focus on the studies by use of synchrotron X-rays to clarify the structures and their dynamical behaviors of polymeric materials. Most of the articles are the out-come of

Kazuo Sakurai

sakurai@kitakyu-u.ac.jp

1 Faculty of Environmental Engineering, The University of Kitakyushu, Fukuoka, Japan

2 Institute for Chemical Research, Kyoto University, Uji, Japan

3 Department of Applied Chemistry and International Institute for Carbon-Neutral Energy Research (WPI-I2CNER), Kyushu University, Fukuoka, Japan academic-industrial collaborations, under the Advanced Softmaterial Beamline Consortium, which is commonly known as Frontier Soft Matter Beamline (FSBL). We wish that this characteristic issue will show an interesting as well as profound aspect of polymers, similarly to the success of the preceding special issues [3-9].

\section{Compliance with ethical standards}

Conflict of interest The authors declare that they have no conflict of interest.

\section{References}

1. Masunaga $\mathrm{H}$, et al. Multipurpose soft-material SAXS/WAXS/ GISAXS beamline at SPring-8. Polym J. 2011;43:471-7.

2. Ogawa $\mathrm{H}$, et al. Experimental station for multiscale surface structural analyses of soft-material films at SPring-8 via a GISWAX/ GIXD/XR-integrated system. Polym J. 2013;45:109-16.

3. Kato T, Aoshima S, Kikuchi H. Special issue: Self-assembled materials. Polym J. 2012;44:451.

4. Asakura T, Asano A. Preface to the special issue: NMR of polymers: recent advances and innovations. Polym J. 2012;44:733.

5. Kanaya T, Sakurai K, Takahara A. Special issue: Application of quantum beams to polymer science and engineering. Polym J. 2013;45:2.

6. Serizawa T. Special issue: Biorelated polymers and materials. Polym J. 2014;46:435.

7. Kato T, Kikuchi H, Imai H. Special issue: Fusion materials: creative development of materials and exploration of their function through molecular control. Polym J. 2015;47:77.

8. Tanaka K, Serizawa T, Chen W-C, Char K, Kato T. Special issue: Polymer surfaces, interfaces and thin films. Polym J. 2016;48:323.

9. Kato T. 50th volume anniversary of Polymer Journal. Polym J. 2018;50:1. 